\title{
Quality improvement initiatives for hospitalised small and sick newborns in low- and middle-income countries: a systematic review
}

Nabila Zaka', Emma C. Alexander², Logan Manikam, ${ }^{1,}$, Irena C. F. Norman², Melika Akhbari², Sarah Moxon Pavani Kalluri Ram ${ }^{5,6}$, Georgina Murphy ${ }^{7}$, Mike English ${ }^{7}$, Susan Niermeyer ${ }^{8,6}$ and Luwei Pearson ${ }^{1}$

\begin{abstract}
Background: An estimated 2.6 million newborns died in 2016; over $98.5 \%$ of deaths occurred in low- and middleincome countries (LMICS). Neonates born preterm and small for gestational age are particularly at risk given the high incidence of infectious complications, cardiopulmonary, and neurodevelopmental disorders in this group. Quality improvement (QI) initiatives can reduce the burden of mortality and morbidity for hospitalised newborns in these settings. We undertook a systematic review to synthesise evidence from LMICs on QI approaches used, outcome measures employed to estimate effects, and the nature of implementation challenges.

Methods: We searched Medline, EMBASE, WHO Global Health Library, Cochrane Library, WHO ICTRP, and ClinicalTrials.gov and scanned the references of identified studies and systematic reviews. Searches covered January 2000 until April 2017. Search terms were "quality improvement", "newborns", "hospitalised", and their derivatives. Studies were excluded if they took place in high-income countries, did not include QI interventions, or did not include small and sick hospitalised newborns. Cochrane Risk of Bias tools were used to quality appraise the studies.

Results: From 8110 results, 28 studies were included, covering 23 LMICs and 65,642 participants. Most interventions were meso level (district and clinic level); fewer were micro (patient-provider level) or macro (above district level). In-service training was the most common intervention subtype; service organisation and distribution of referencing materials were also frequently identified. The most commonly assessed outcome was mortality, followed by length of admission, sepsis rates, and infection rates. Key barriers to implementation of quality improvement initiatives included overburdened staff and lack of sufficient equipment.

Conclusions: The frequency of meso level, single centre, and educational interventions suggests that these interventions may be easier for programme planners to implement. The success of some interventions in reducing morbidity and mortality rates suggests that Ql approaches have a high potential for benefit to newborns. Going forward, there are opportunities to strengthen the focus of Ql initiatives and to develop improved, larger-scale, collaborative research into implementation of quality improvement initiatives for this high-risk group.
\end{abstract}

Trial registration: PROSPERO CRD42017055459.

Keywords: Newborns, Quality improvement, Low- and middle-income countries, Systematic review, Preterm, Neonatal mortality rates, Infection control, Hospital stay

\footnotetext{
* Correspondence: logan.manikam.10@ucl.ac.uk

'UNICEF New York, UNICEF House, 3 United Nations Plaza, New York, NY

10017, USA

${ }^{3} \mathrm{UCL}$ Institute Epidemiology \& Healthcare, 1 - 19 Torrington Place, London

WC1E 6BT, UK

Full list of author information is available at the end of the article
}

(c) The Author(s). 2018 Open Access This article is distributed under the terms of the Creative Commons Attribution 4.0 International License (http://creativecommons.org/licenses/by/4.0/), which permits unrestricted use, distribution, and reproduction in any medium, provided you give appropriate credit to the original author(s) and the source, provide a link to the Creative Commons license, and indicate if changes were made. The Creative Commons Public Domain Dedication waiver (http://creativecommons.org/publicdomain/zero/1.0/) applies to the data made available in this article, unless otherwise stated. 


\section{Background}

Newborns, or infants under 28 days of age, account for the greatest proportion of deaths amongst underfive children. The vast majority of these deaths occur in low- and middle-income countries (LMICs), which in 2016 comprised $98.5 \%$ of the 2.61 million neonatal deaths worldwide [1]. The estimated average of neonatal mortality rates in LMICs was 20 per 1000 live births, compared to 3 per 1000 in high-income countries [1]. Targeting this high-risk group is thus an urgent policy priority, particularly regarding the three major causes of neonatal deaths, which are preterm birth complications, severe infections, and intrapartum complications [2].

Low birth weight newborns (birthweight of $<2500 \mathrm{~g}$ ) are especially vulnerable. Newborns can have low birth weight due to prematurity and/or being small for gestational age (SGA). A neonatal mortality relative risk analysis found that preterm and SGA babies had relative risks of 6.82 and 1.83, respectively, compared to nonlow birth weight infants [3]. Survivors of premature birth are at risk of cardiovascular dysfunction, chronic lung disease of prematurity, neurodevelopmental problems, and visual and sensory impairment [4]. SGA newborns likewise have a higher risk of stunting and later, cardiac, metabolic, and cognitive impairment (although to a lesser extent than preterm babies) $[5,6]$. Babies who become ill also suffer severe consequences-meta-analysis of newborns in sub-Saharan Africa, South Asia, and Latin America estimated an incidence risk of $7.6 \%$ for possible severe bacterial infection, with an associated case-fatality risk of $9.8 \%$ [7]. Intrapartum complications can result in neonatal encephalopathy and longer term consequences of disability and cerebral palsy [8].

Most small and sick newborns require inpatient hospital care, ideally in a dedicated unit, and there is a great potential for quality improvement (QI) in this setting, especially in LMICs. Quality improvement is defined by Ovretveit as "better patient experience and outcomes achieved through changing provider behaviour and organisation through using a systematic change method and strategies" [9]. Change of behaviour and organisation can occur at different levels, for example, micro interventions, at the level of the patient-provider interaction (e.g., distribution of referencing materials to providers); meso interventions, at the district and clinic level (e.g., service organisation); or macro, above district level (e.g., regulation and governance) $[10,11]$. The Lancet Every Newborn series estimated that increased coverage and improvements at and around the time of birth could avert $71 \%$ of deaths [12]. Preventing nosocomial infections [13, 14], irrational antibiotic usage [15], inadequate use of Kangaroo Mother Care [16], low adherence to use of breast milk [17], unsafe oxygen use
[18], insufficient bonding with mothers and parents [19], and increasing adherence to humane [20] and baby friendly $[21,22]$ hospital care are all potential areas for QI. As LMICs are increasingly committing to plan and invest in hospital infrastructure and capacity building of health providers for small and sick newborn care, it is timely to strategise and support simultaneous quality improvement efforts.

Previous reviews that have touched on QI initiatives for newborns in LMICs have focused on maternal and child health outcomes together. Dettrick et al. found that evidence in this area is often poor, and most research focuses on service utilisation above other indicators [23]. Additionally, Althabe et al. conducted a review of systematic reviews [24]. Amongst reviews covering over 300 studies, only 18 of these were conducted in LMICs, illustrating the disparity in the amount of research undertaken in these countries [24]. Austin et al. examined approaches to improve maternal and newborn care across all types of countries and observed that quality improvement interventions in LMICs have tended to be community based, rather than hospital based [25].

This review seeks to contribute to the existing evidence base by synthesising data on quality improvement initiatives for hospitalised small and sick newborns in LMICs. We aim to address the following objectives within eligible studies:

- To identify and categorise quality improvement approaches for small/sick hospitalised newborns in LMICs

- To identify and categorise outcomes investigated by quality improvement initiatives for small/sick hospitalised newborns in LMICs

- To identify barriers and promoters, at a local level and systems level, to the implementation of quality improvement initiatives for small/sick hospitalised newborns in LMICs

Our work will serve as a guide to quality improvement initiatives in this area by synthesising evidence on approaches used, outcome measures employed to estimate effects, and the nature of implementation challenges, for the information of future healthcare workers undertaking similar initiatives which should be evidence-based.

\section{Methods}

A protocol for this review was published on the PROSPERO register in January 2017, registration number CRD42017055459 (PROSPERO, 2017).

\section{Eligibility criteria}

Studies were included if they met the following criteria: 
- Populations: hospitalised small and/or sick newborns in LMICs and admitted for inpatient healthcare. LMICs were identified according to the World Bank list of LMICs [26]. Facilities for this population must be defined as 'hospitals' or units within hospitals.

- Interventions: quality improvement initiatives, according to the Ovretveit definition-"better patient experience and outcomes achieved through changing provider behaviour and organisation through using a systematic change method and strategies" [9].

- Outcomes: objective clinical outcomes relating to mortality, morbidity, and process of care measures.

- Language: studies published in English, or with translation available.

- Year: published from 2000 or later.

The year cut-off was chosen in order to focus the review on recent practice in the context of changing healthcare systems. Studies that focused solely or primarily on practices in the delivery room that encompassed small/sick newborns only as a subset, or studies that focused on community interventions, were deemed not to meet the eligibility criteria of hospitalised small and sick newborns. Outcomes of self-assessed competency, or patient-assessed satisfaction, were also deemed not to meet the eligibility criteria of clinical outcomes, as this method of assessment was seen to be an insufficient proxy for an objectively measured clinical outcome. Finally, in order to simplify the review, studies focusing only on implementation of Kangaroo Mother Care (KMC), a specific method of care for preterm infants that focuses on encouraging skin-to-skin contact between mother and infant, were not included unless the study involved modification to KMC implementation, because KMC has been well covered in systematic reviews elsewhere [27, 28].

\section{Information sources}

We searched the following electronic databases from January 2000 onwards: Medline, EMBASE, WHO Global Health Library, and Cochrane Library. We searched the trial registries: WHO ICTRP and ClinicalTrials.gov for completed and ongoing studies. Searches were conducted in April 2017. The literature searches of peerreviewed publications were supplemented by scanning the reference lists of relevant studies and systematic reviews. Interlibrary lending was used to access certain papers.

\section{Search strategy}

The following search strategy was designed to capture studies that were suitable for inclusion in the review. For example, the search strings used in EMBASE were:
Term 1: Quality improvement

(Quality or performance or effectiveness) AND (care or improvement* or increase* or service\$ or indicator\$)

Term 2: Newborns

Neonat" or neo-nat* or Baby or Babies or Newborn\$ or new-born\$ or infant\$

Term 3: Hospitalised

Inpatient\$ or in-patient\$ or hospitalis* or NICU or neonatal intensive care unit.

These search terms were then combined to give a final search of Term 1 AND Term 2 AND Term 3, which was used to search abstracts in these databases.

\section{Study selection, extraction, and analysis}

The titles and abstracts were screened by two researchers independently for inclusion/exclusion, with disagreements resolved with arbitration from a third reviewer. The results shortlisted for inclusion and then underwent full-text screening, again undertaken by two researchers independently with arbitration from a third, to produce a final shortlist of articles to be included in the review. Data were extracted from each paper by one researcher and checked by a second, using a piloted worksheet, the details of which are supplied in Additional file 1. Summary results from fields that are not presented in the main manuscript are available on request.

To standardise study classifications, the following definitions were used, based on NICE definitions [29]:

1. Randomised controlled trial-similar people are allocated, at random, to different groups in order to test the efficacy of an intervention, with one group receiving the tested intervention

2. Intervention study (non-random)-similar people are allocated, via a non-random process, to different groups in order to test the efficacy of an intervention, with one group receiving the tested intervention

3. Before and after study—dependent variables are assessed in a setting before and after an intervention is applied, where the population may be the same or differ

QI approaches were classified according to the Kruk and Gage schema 'Synthesizing improvement approaches' [10]. This classifies approaches at the micro, meso, or macro level, meaning at the level of the patient-provider interaction, such as on the sick newborn care unit; at the district and clinic level; at an individual hospital; or at the above district level, such as across a health system. It then provides subclassifications of approaches within each of these classes. 
The relevant outcomes of the studies were extracted. Each outcome was then classified by a research team member. These classifications were based on the WHO-defined components of 'quality of care' [30]. This states that high-quality care should be Safe, Effective, Timely, Efficient, Equitable, and Peoplecentred. Studies were sub-sorted by results, according to whether they reported that there was a significant increase in the metric during their study, a significant decrease, no significant change, or if statistical significance was not assessed or not reported. Significance was defined as either $p<0.05$ or using the $95 \%$ confidence interval.

Barriers and promoters of quality improvement were classified as local level, meaning individual to the particular hospital or location where the intervention was based, and systems level, meaning a factor that would necessarily influence hospitals and locations beyond the local area. We utilised a wide scope for extraction of barriers and promoters, including both barriers and promoters that were specific objects of study and those that were informally reported such as through staff feedback reported in the discussion.
In order to assess bias in the included studies, we utilised the Cochrane Risk of Bias for Non-Randomised Studies of Interventions (ROBINS-I) and revised tool for Risk of Bias in randomised trials (RoB 2.0) to assess included quantitative studies [31, 32]. Any study with a Critical ROBINS-I Overall Bias or a High RoB 2.0 Overall Bias classification was omitted from the "Results" and "Discussion" sections of this review. Two independent reviewers conducted the quality appraisal, and adjudication was provided by a third reviewer if warranted.

\section{Results}

\section{Study selection}

In total, the searches returned 8110 results across the four databases, with 49 results identified from other sources. After 2228 duplicates were excluded, a total of 5931 results' titles and abstracts were screened against inclusion criteria. Of these, 5677 results were excluded for not meeting inclusion criteria at this stage, as demonstrated by Fig. 1 .

This left 254 papers, which underwent full-text review. As shown in Fig. 1, the most common reason for exclusion at this stage was that studies took place in countries

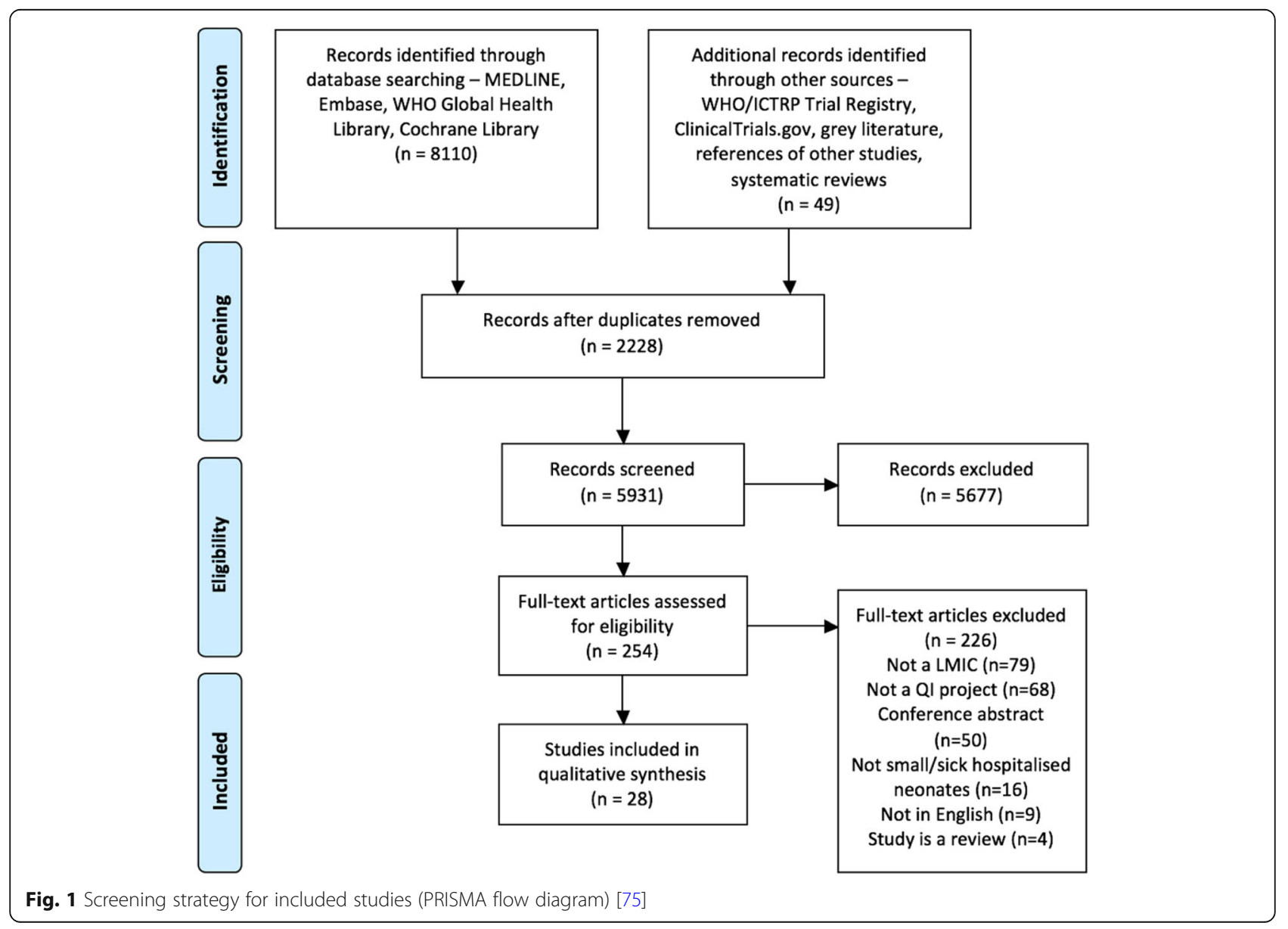


that were not LMICs $(n=79)$ according to the World Bank classifications [26]. The second most common reason was that studies were not describing QI interventions $(n=68)$. Twenty-eight studies were ultimately identified as suitable for inclusion in the narrative synthesis. Owing to the heterogeneity of QI methodologies, settings, and outcomes measured, meta-analysis was not undertaken.

\section{Study and participant characteristics}

The 28 studies consisted of 25 controlled before and after studies, two intervention studies (non-random), and one randomised controlled trial. Table 1 provides a summary of all the included studies.

The 28 included studies included data from 23 different countries. This included data from India (4 studies), China (3 studies), Bangladesh (3 studies), Ghana (3 studies), Brazil (3 studies), Iran (2 studies), El Salvador (2 studies), Mexico (2 studies), the Philippines (2 studies), Tunisia (2 studies), Turkey (2 studies), Uganda, Nepal, Kenya, Guatemala, Mozambique, Lebanon, Sierra Leone, Pakistan, Argentina, Colombia, Peru, and Morocco. The review includes two multi-country analyses, with one covering Argentina, Colombia, El Salvador, India, Mexico, Morocco, Peru, the Philippines, Tunisia, and Turkey and the other covering El Salvador, Mexico, the Philippines, and Tunisia [33, 34].

There were a total of 65,642 reported participants included in analyses across the studies, although one large study included 29,508 deliveries in its analysis and another included 15,249 [35, 36].

Table 2 presents the ROBINS-I and RoB 2.0 ratings awarded to each of the studies. One study scored Critical in Overall Risk of Bias, and thus was excluded from data synthesis, but is included in Tables 1 and 2 [37].

The core narrative themes extracted from the papers are presented under the headings, classified QI approaches, groups of outcomes measured in QI approaches, and barriers and promoters to implementing QI approaches. Henceforth, summary data comes from the 28 studies with Overall Risk of Bias of Serious or lower.

\section{Classified quality improvement approaches}

We categorised the overarching approaches for quality improvement used for sick newborn care using the Kruk and Gage 'Synthesizing improvement approaches' schema [10]. We found 11 studies with micro interventions [33, 38-47], 23 studies with meso interventions [34-36, 38-42, 44-46, 48-59], and two studies with macro interventions $[59,60]$. Nine of the studies had mixed-level interventions, with eight of these being meso and micro and one being macro and meso.
Table 3 provides information on approaches for quality improvement by subtype. In addition to the included subtypes in Table 3, there were additional categories according to the Kruk and Gage schema, for which no studies utilised those particular methods; these were, at the macro level, pay for performance, other financing and incentives, pre-service training, and external to health system and, at the meso level, mortality audits and social franchising [10].

The most frequent subtype of intervention was the meso approach 'In-service training', used by 20 studies. Such interventions were often delivered as part of a group of innovations-for example, Rosenthal et al. introduced a multifaceted infection control bundle incorporating education on hand hygiene and asepsis, and Agarwal et al. developed a package of interventions that included on-job training of nurses in common neonatal skills $[34,36]$. The intervention of Clark et al. was based on a WHO Emergency Triage and Treatment training course, and Sethi et al. utilised a Comprehensive Post-Natal Counselling package, comprised of education of health care providers and family members [50, 54]. In Zhou et al., key staff members attended a training workshop run by the Canadian Neonatal Network for 2 days, and attendees in turn then trained the other Neonatal Intensive Care Unit (NICU) team members [46].

The second most frequent subtype of intervention was the meso approach, 'Service organisation', used by nine studies. For example, Rahman et al. described an approach that involved service reorganisation with triage and fast assessment and use of a record system [44]. Erdeve et al. evaluated the impact of individual rooms on patients and families in the NICU [52].

The most frequent micro approach was 'Distribution of Referencing Materials to Providers' by eight studies. Pinto et al. disseminated a new protocol proposed by the Brazilian National Health Surveillance Agency for antibiotic usage in low birth weight infants [43]. Salehi et al. also described the implementation of new guidelines, as did Mais et al. and Gathara et al. as part of their approaches [39, 41, 45].

Two studies utilised macro approaches, which were regulation and governance, and task shifting. Ramaswamy et al. used regulation and governance in their development of the Ridge-Kybele Model for Obstetric and Neonatal Care, an integrated approach for systems change which prioritises capacity building in order to properly embed change practices [59]. Yawson et al. utilised task shifting, by using a tool to identify service gaps which led to national and regional operating plans being developed and implemented to improve neonatal care [60]. 


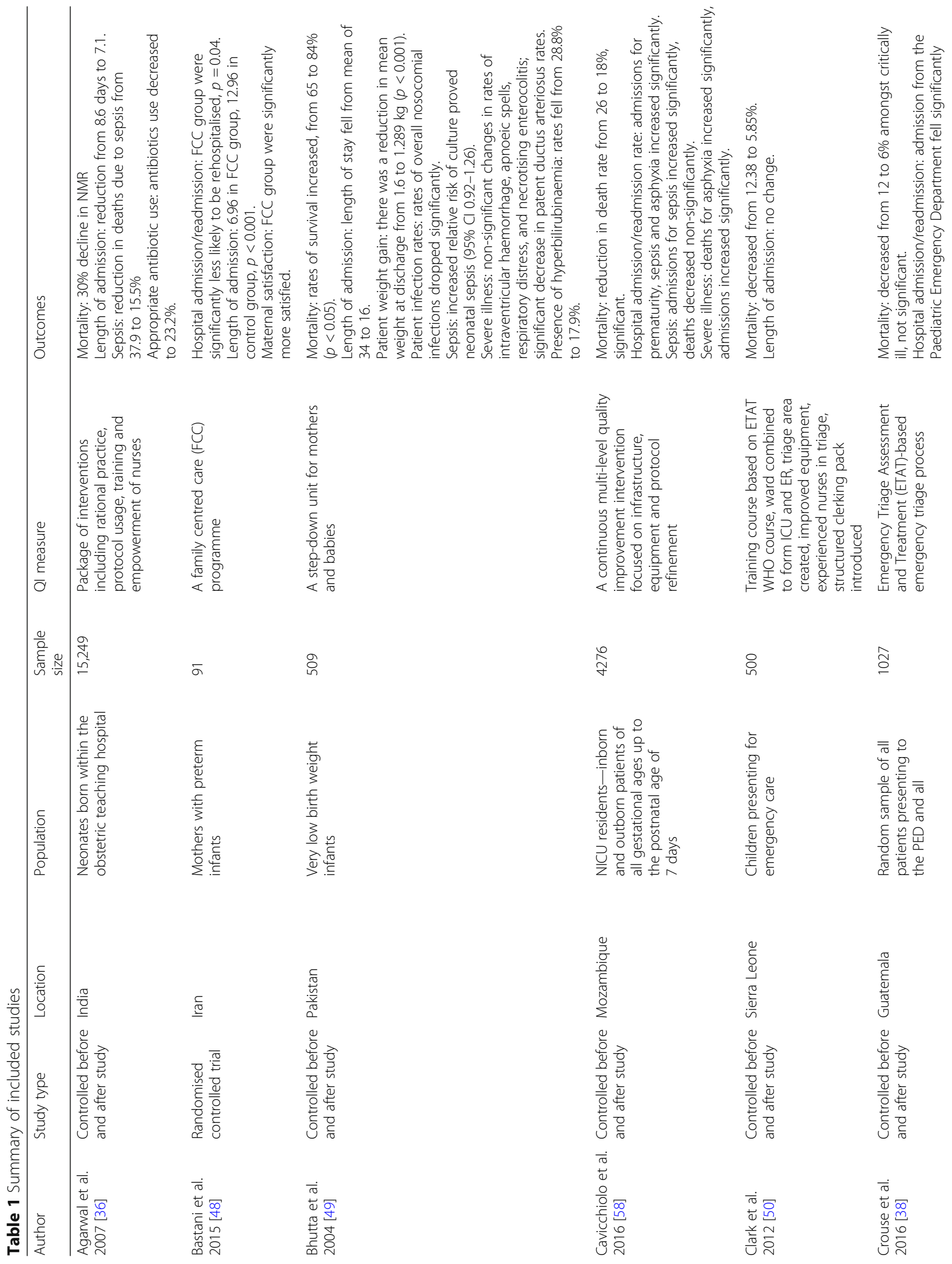




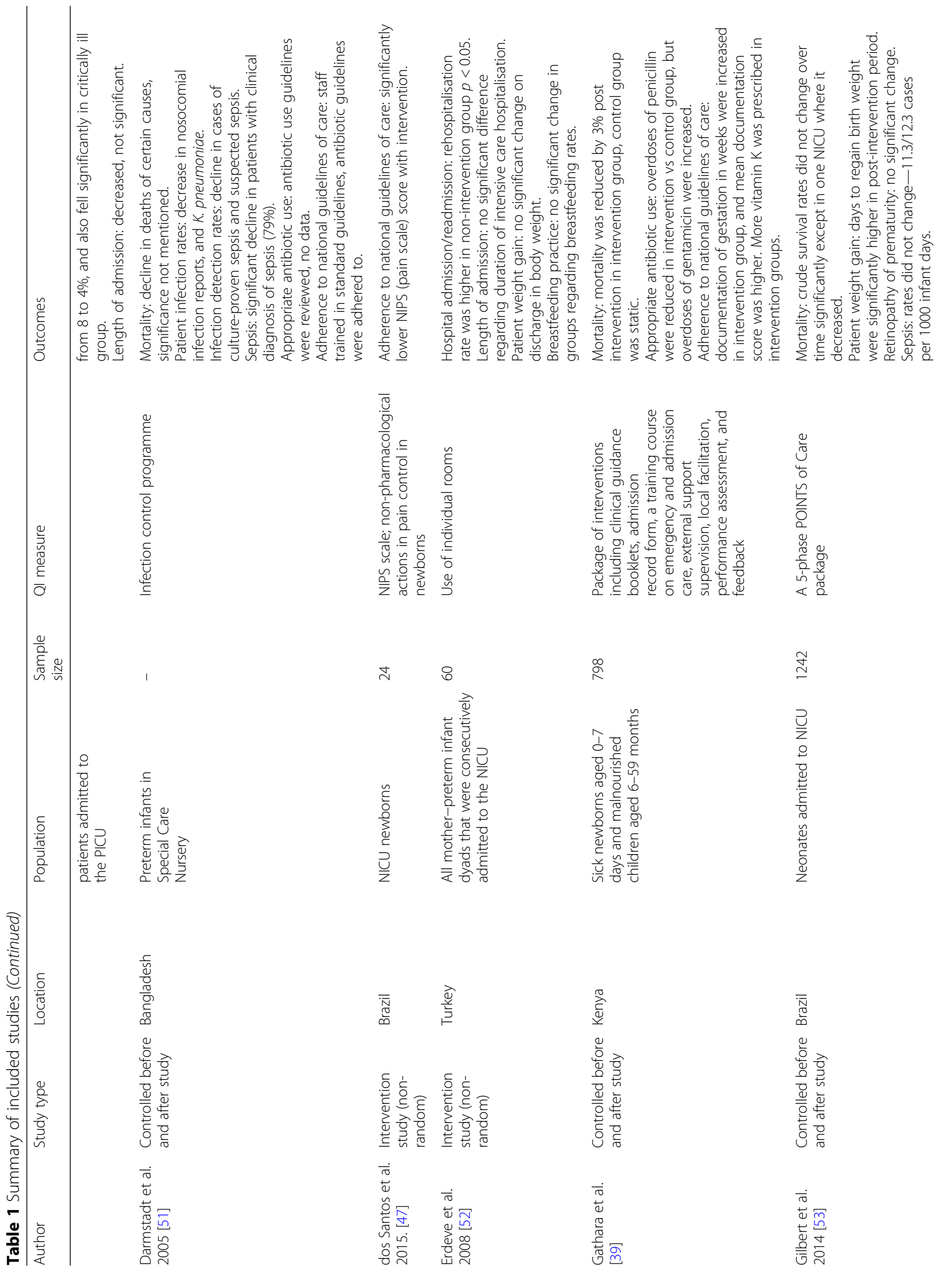




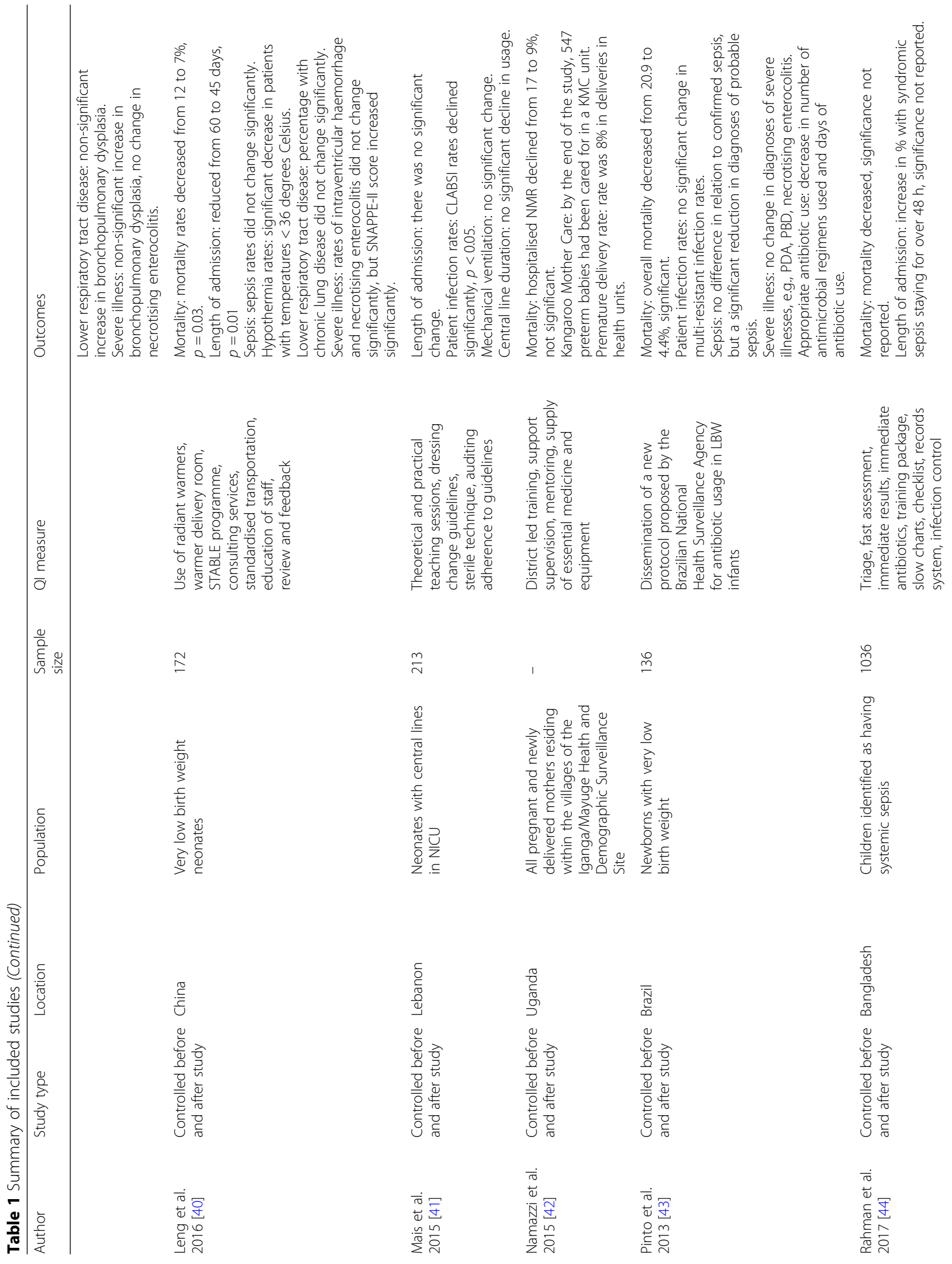




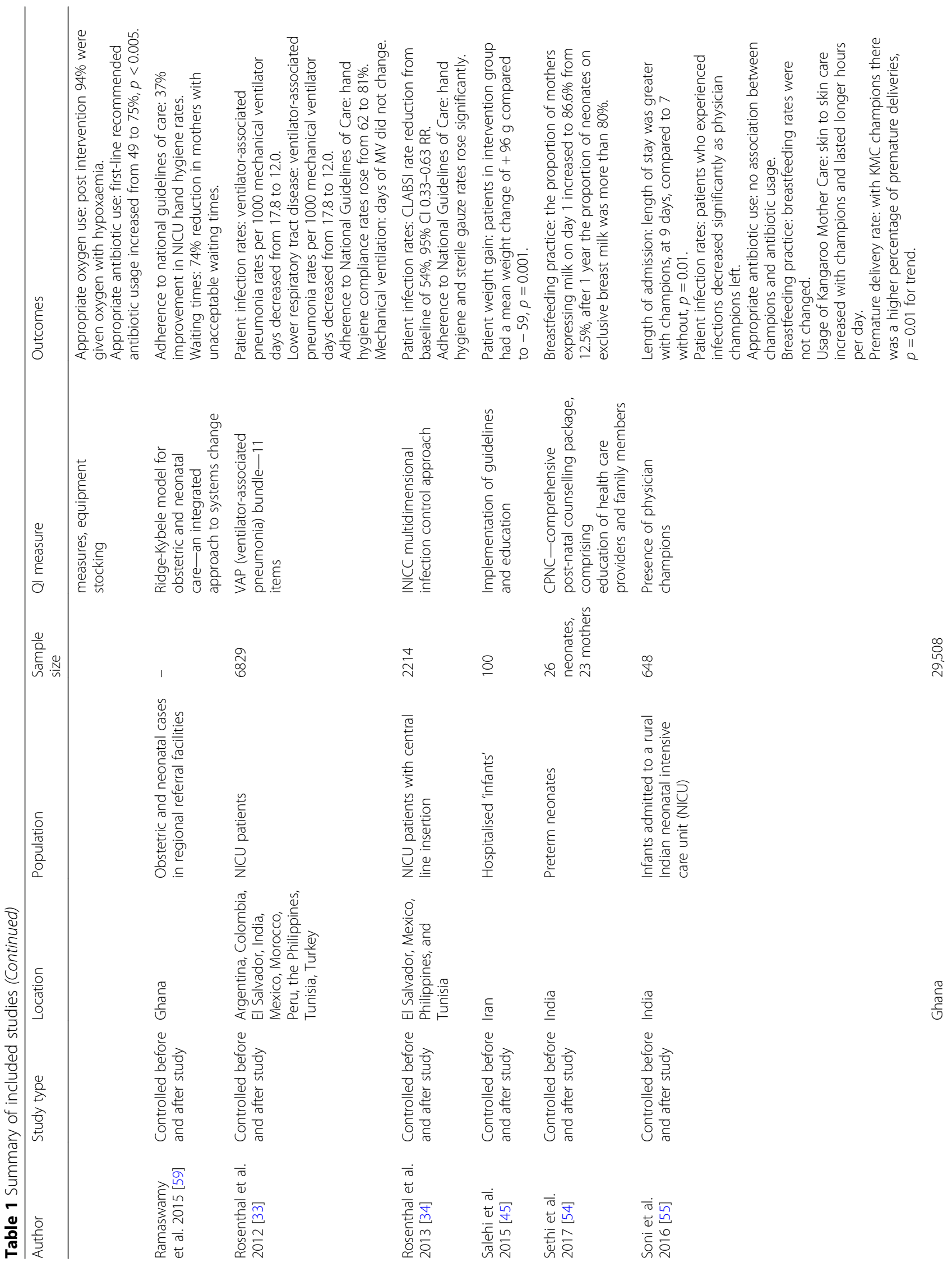




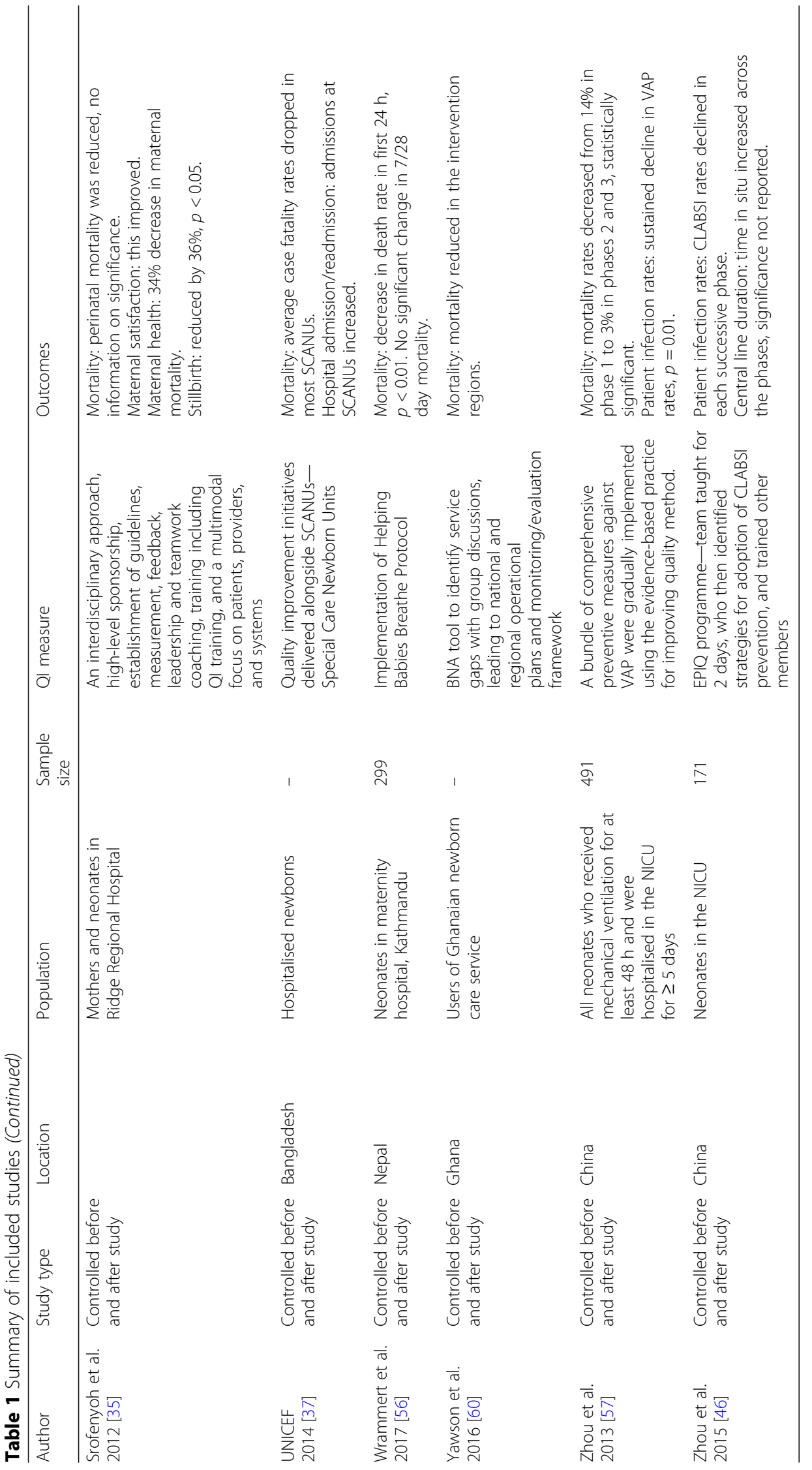




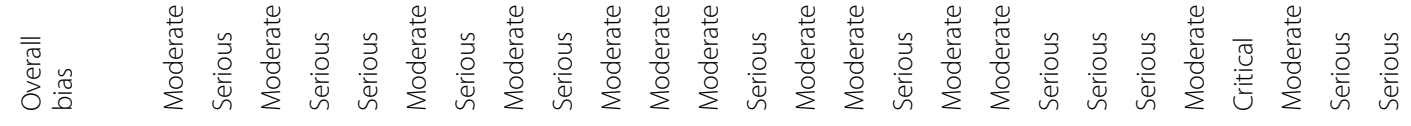

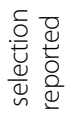

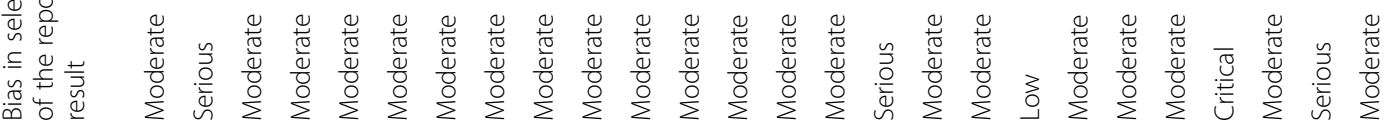

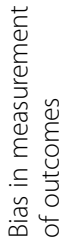

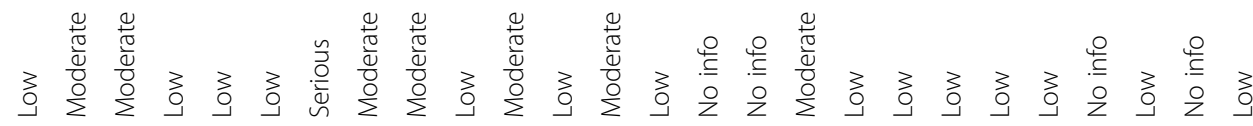

$\circ \frac{\pi}{\frac{\pi}{0}}$

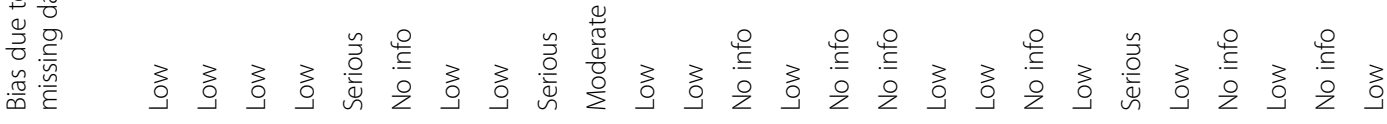

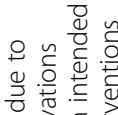

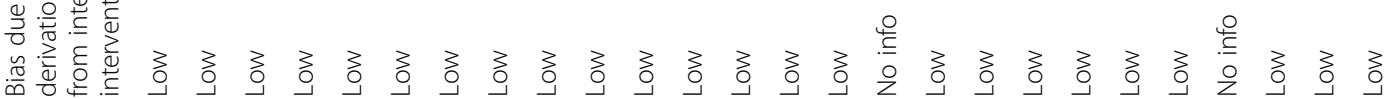

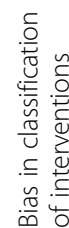

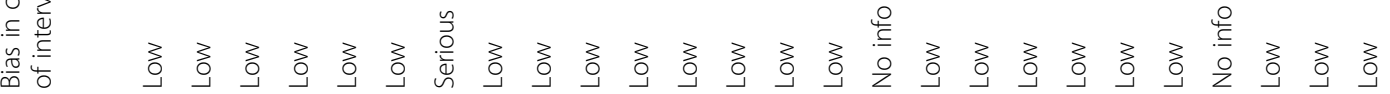

4

읍. 올

过

늘 을

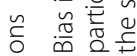

药

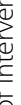

맘

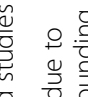

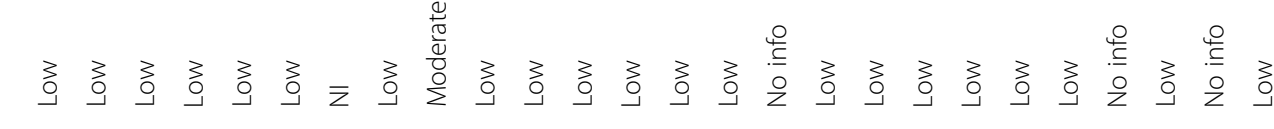

㻤

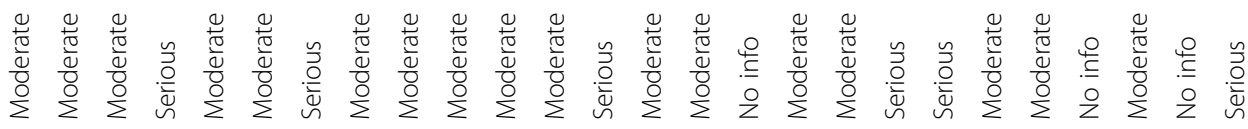




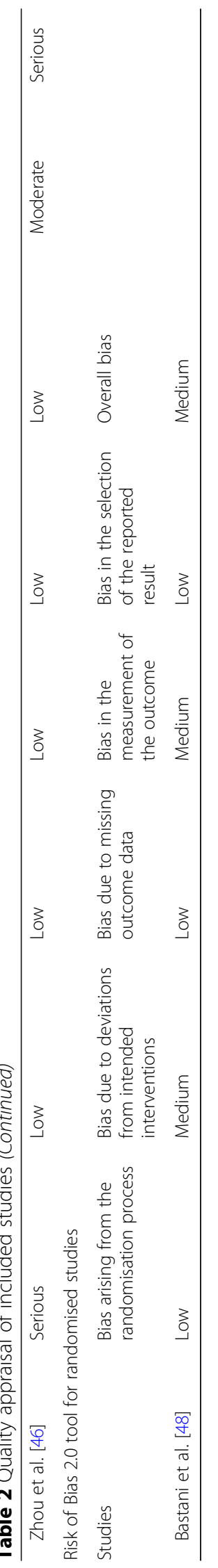


Table 3 Subtype of intervention

\begin{tabular}{llll}
\hline Level & Strategy & Total & Citation \\
\hline Micro & Distribution of referencing materials to providers & 8 studies & {$[33,38,39,41,43-46]$} \\
& Decision support & 2 studies & {$[39,40]$} \\
Meso & Care coordination & 5 studies & {$[33,39,42,46,47]$} \\
& Strengthening facility infrastructure & 6 studies & {$[35,42,44,50,57,58]$} \\
& Continuous quality improvement & 7 studies & {$[34,35,41,46,51,58,59]$} \\
& Supervision & 5 studies & {$[35,39,51,55,57]$} \\
& Feedback & 6 studies & {$[34,35,39,40,51,59]$} \\
& In-service training & 20 studies & {$[34-36,38-42,44-46,48,50,51,53,54,56-59]$} \\
& Service organisation & 9 studies & {$[35,36,39,40,44,49,50,52,59]$} \\
& Regulation and governance & 1 study & {$[59]$} \\
\hline
\end{tabular}

Groups of outcomes measured in quality improvement approaches

A total of 23 broad outcomes were used to assess the efficacy of the QI interventions, shown in Table 4. The majority of these, 13, were outcomes that were classified as aiming at delivering safe care, defined as "delivering health care which minimises risks and harm to service users, including avoiding preventable injuries and reducing medical errors" [30]. Five were aimed at delivering effective care, two efficient care, two people-centred care, and one timely care. No studies were identified as including outcomes addressing the delivery of equitable care.

\section{Mortality rate}

Mortality rate was the most frequently measured outcome, assessed by 16 studies. Studies used a variety of mortality metrics, including 28-day mortality, mortality within the study period, and specific cause mortality. Eight studies found that introduction of the QI intervention was associated with a significant decrease in mortality, four found no significant change, and five others reported results but not the statistical significance of those results. Amongst the more successful interventions, Bhutta et al. found that survival in their NICU increased from 65 to $84 \%, p<0.05$, after a policy change to create a stepdown unit and involve mothers earlier in the care of their at risk infants [49]. Pinto et al. also found a significant reduction in mortality, from 20.9 to $4.4 \%$, $p=0.009$, after the dissemination of a new antibiotic protocol to their NICU, which was supervised by two neonatologists in charge of the clinical routine [43]. Leng et al. also found mortality rates decreased, from 12 to $7 \%, p=0.03$, amongst newborns transferred from eight hospitals to their Level III NICU, after the introduction of a package of interventions including the STABLE programme and staff education [40]. Crouse et al. found that overall mortality for the critically ill patients decreased with their new emergency triage process, from $12.4 \%$ pre-intervention to $6.0 \%$ postintervention, but this was not statistically significant $(p=0.15)$ [38].

\section{Length of admission}

Length of admission was the second most assessed outcome, by ten studies. Studies reported mixed outcomes, with one reporting a significant increase, four a significant decrease, two no change and three where significance was not assessed. Soni et al. found that length of stay in the NICU increased with identified Kangaroo Mother Care champions, at a median of 9 days, compared to a median of seven without the champions [61]. Conversely, Bastani et al. found that, in their RCT, mean length of stay in the NICU for their family-centred care group was 6.96 days compared to 12.96 in the control group, $p<0.001$ [48]. Bhutta et al. found that length of stay more than halved after their stepdown unit was introduced, from a mean of 34 days pre-intervention to 16 postintervention [49].

\section{Sepsis rates}

Sepsis rates were the joint third most frequently measured outcome, assessed by eight studies. Three studies reported a significant decrease in sepsis rates, and five reported no significant change. Amongst the studies that reported significant decreases were Agarwal et al., who found that there was a severe reduction in deaths in their neonatal unit due to sepsis after the introduction of their multi-faceted intervention package, from $37.9 \%$ pre-intervention to $15.5 \%$ post, $p<0.01$ [36]. Their package included greater involvement of mothers in caregiving, as with Bhutta et al., alongside enforced aseptic routines, greater use of protocols, education, and other features [36, 49]. 


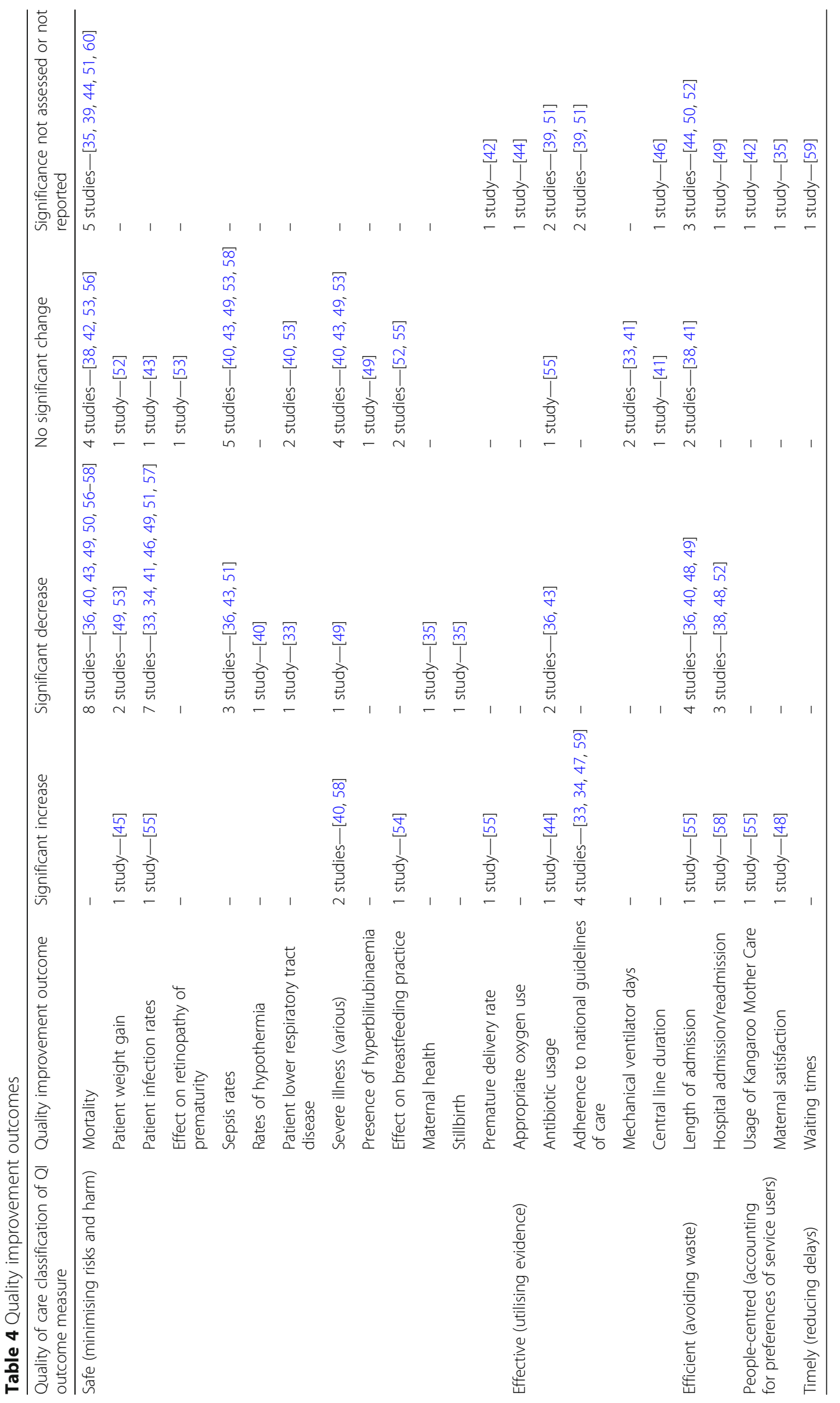


However, in Gilbert et al., the introduction of an educational package, POINTS of Care, did not change sepsis rates across five neonatal units nor did the multi-faceted intervention incorporating education, feedback, and other elements in Leng et al. [40, 53].

\section{Patient infection rates}

Patient infection rates, across a variety of different specific infections, were assessed by nine studies. Seven studies reported a significant decrease in infection rates, one a significant increase and one reported no significant change. Amongst the studies that reported significant decreases was Rosenthal et al., who found that a multi-faceted ventilator-associated pneumonia (VAP) bundle intervention was associated with a reduction in VAP rates from $17.8 / 1000$ ventilation days pre-intervention to $12.0 / 1000$ ventilation days post-intervention, across 15 NICUs in ten countries [33]. Rosenthal et al. found that after an infection control bundle, central line-associated bloodstream infection (CLABSI) rates reduced across four NICUs in four countries, resulting in a relative risk of 0.45 postintervention (95\% CI 0.33-0.63) [34]. Both interventions utilised infection control teams and surveillance. Mais et al. also reported on CLABSI rates, which declined from $15 / 1000$ central line days before the introduction of a bundle of interventions to 6.4/1000 afterwards $(p<0.05)$ [41]. For Zhou et al., CLABSI rates also fell significantly from $16.7 / 1000$ before the introduction of a nursing training programme to $5.2 / 1000$ afterwards [46].

\section{Barriers and promoters to implementing quality improvement approaches}

Many factors have the potential to either promote or inhibit the successful implementation of interventions for quality improvement $[62,63]$, and several of these were covered by the studies. These factors are noted in Table 5 and are identified as either barriers or promoters operating at a local or systems level. In total, 11 barriers were identified, with six at the local level and five at the systems level, and 13 promoters were identified, with nine at the local level and four at the systems level. No study assessed the statistical significance of any barrier or promoter; identified barriers and promoters were largely inductive by the investigators.

\section{Promoters}

Nine promoters were identified at the local level by seven studies. Three studies highlighted the importance of motivation of key individuals. Darmstadt et al. highlighted the will and effort of key individuals as being important, especially the nursing supervisor; Srofenyoh et al. and Namazzi et al. noted the importance of local champions $[35,42,51]$.
Four promoters were identified at the systems level by four studies. Yawson et al. stated that good quality national data collection is essential for designing QI interventions [60]. Namazzi et al. highlighted structured community relationships, Cavicchiolo et al. said NGO collaboration could be helpful, and Srofenyoh et al. valued formal support from the Ghanian Health Service [35, 42, 58].

\section{Barriers}

Six barriers to quality improvement initiatives were identified at a local level by eight studies. The barrier posed by overburdened staff was the joint most frequently mentioned barrier, by four studies. This includes Gilbert et al. who mentioned staff being overstretched as an impediment, and Namazzi et al. who discussed the fact that facility staff had competing demands on their time [42, 53]. Four studies also mentioned the problem of insufficient equipment, such as Crouse et al. who stated that paper supplies running low and no computerised patient records hampered record keeping [38].

Five barriers were identified at a systems level by four studies. Namazzi et al. highlighted both the problems of increasing demand for services at all hospitals and lack of finances for necessary medicines [42]. Gilbert et al. said government policies enforcing redistribution of staff from study NICUs to underserved areas also created barriers [53].

\section{Discussion}

Many LMICs have focused on developing the infrastructure for inpatient care of sick newborns in public health systems. The recent focus on quality of care with the launch of the Quality, Equity and Dignity Network co-led by the WHO and United Nations International Children's Emergency Fund (UNICEF), alongside ongoing efforts to raise the standard of paediatric care, has increased the interest in addressing gaps in quality of care for sick newborns. To our knowledge, this is the first systematic review to specifically examine quality improvement initiatives for hospitalised small and sick newborns in LMICs. Previous reviews have focused on maternal and child care as a whole $[23,24]$, or did not focus on LMICS individually [25], or have focused on a subtype of quality improvement interventions (in service training) [64], with all of these also encompassing nonhospitalised newborns.

\section{Programmatic implications}

Programme planners should consider that the majority of interventions are at the meso level, and many studies (20 studies) involved in-service training as part of their 


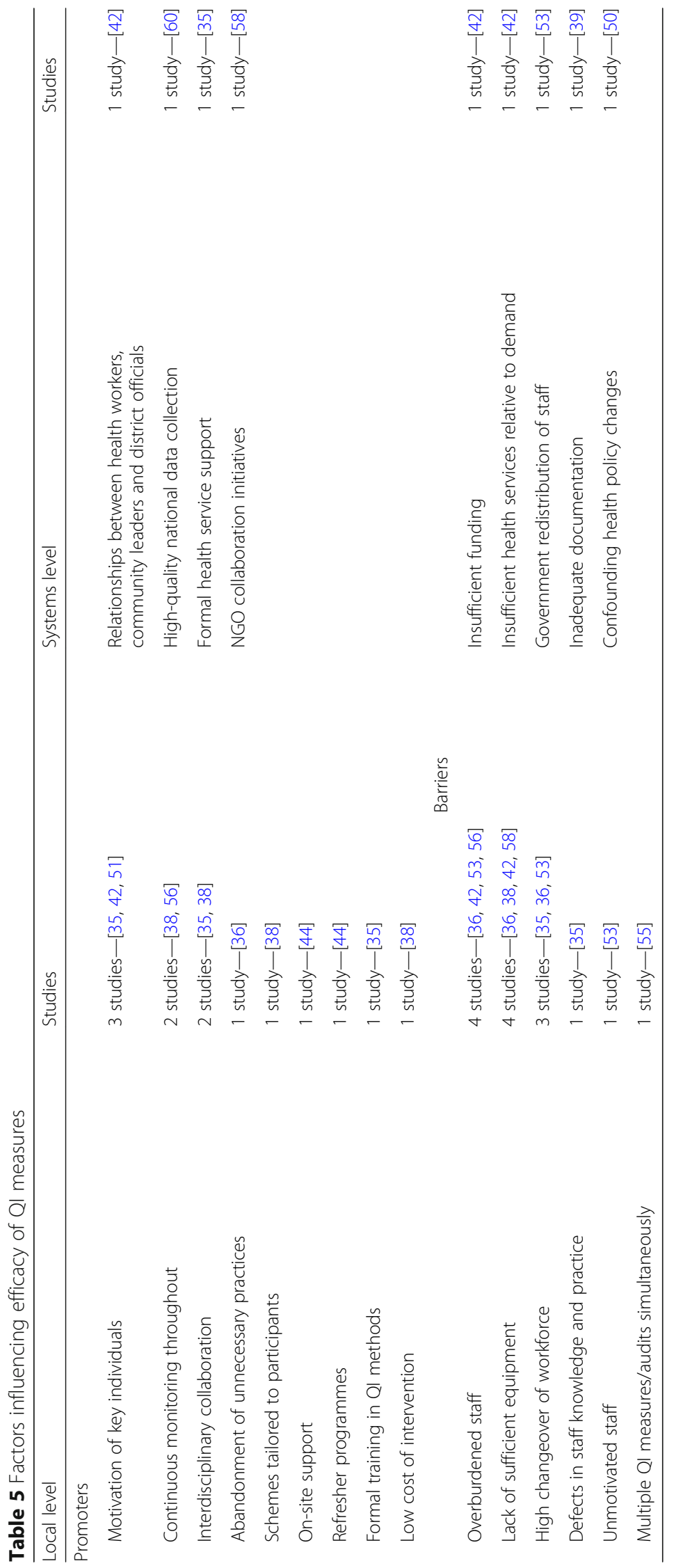


interventions. Educational interventions may be frequently represented because exposure of the intervention to the relevant staff can be appropriately monitored, implemented, and tailored to local needs, whereas macro-level interventions like regulation and governance or task shifting require greater continuous coordination. For planners designing their first QI projects, in-service training may be an advisable first step. However, structural adjustments remain necessary in order to facilitate QI at all levels of care. It will be especially relevant to formulate and implement policies to retain skilled nursing staff alongside providing financing to achieve national standards of minimum infrastructure and equipment, and to increase staffing for optimal nursepatient ratios. Increasing adoption of perinatal death reviews in LMICs will also provide an opportunity to review and address common gaps in a country through macro-level interventions.

The most frequently assessed outcomes were mortality rates, sepsis and infection rates. Eight of the 12 studies that investigated statistically significant differences in mortality rates observed a statistically significant decrease in mortality. Particularly high reductions occurred in Bhutta et al. and Pinto et al., with mortality falling by $19 \%$ in the former after the creation of a stepdown unit and mortality falling by $16.5 \%$ in the latter after the dissemination of a new antibiotic protocol [43, 49]. These studies demonstrate the potential for QI to produce swift and significant benefits for this vulnerable patient group. However, it is important to note that the majority of the included studies were non-randomised beforeafter studies, and a review by Schouten et al. found that observational studies tend to demonstrate larger effects than more rigorous designs [65]. There are many feeders into mortality rates and a number of the studies had important confounders. With regards to other outcomes, in general, studies did not report greater involvement of family members as part of their interventions, but those that did reported significant positive results for mortality, sepsis, and readmission rates, suggesting this area could be explored further $[36,48,49]$. It will be important to focus future QI efforts on sepsis due to rising rates of antibiotic resistant infections and sepsis in this group [66]. Some successful studies in this area did incorporate holistic interventions involving family members [36, 49].

Several studies benefitted from focusing their efforts on single pathologies (ventilator-associated pneumonia, central line-associated blood stream infections) for quality improvement, which allowed for collation of findings across multiple centres [33, 34]. Other interventions utilised training courses included the POINTS of Care training (a six module training programme covering topics including pain control and nutrition interventions) [53, 67] and the STABLE programme (a continuing education course that focuses on stabilising sick newborns) [40]. Such approaches allow easy monitoring of attendance and hence exposure for future studies that may choose to utilise control groups.

For programme planners, our identification of barriers and promoters to successful QI interventions will be helpful to accelerate efforts for meeting the objectives of Every Newborn Action Plan, Every Woman Every Child, and other quality of care agendas $[68,69]$. Planners should consider resolving barriers such as overburdened staff, which may be more severe during times of higher seasonal demand, and consider identifying 'staff champions' to promote their projects which were reported as promoters in several studies [35, 42, 51]. A previous review of barriers and enablers of KMC identified similar influential factors, such as the barrier posed by a high workload [70].

Finally, it is important to reflect that quality improvement approaches are heavily dependent on data linked to action and evidence to drive positive change. Moxon et al. have advocated for the urgent need to improve health management information systems and monitoring of hospital care for newborns for better measurement of quality of care and to identify and address quality gaps [62]. Thus, the allocation of resources on improving quality of sick newborn care needs to factor in requirements for improving local, country-led sustainable information systems, as well as systematic use of perinatal death audits [71]. Mortality audits were not utilised by any of the included studies, despite a recent focus via Maternal and Perinatal Deaths Surveillance and Response projects [72]. The bottlenecks of health financing, health workforce, data and community engagement need more varied approaches to implementation and research, especially at Macro level, and advanced information systems would be valuable in order to optimise QI if used for action at the correct level of the health system.

\section{Research implications}

Regarding future research into QI initiatives in LMICs, we recommend QI implementation takes place in tandem with strong data collection and monitoring. The majority of research takes place in high-income countries, whose settings may not be fully applicable to LMICs [12, 25]. We also note the impact of several interventions was hard to assess because studies did not analyse statistical significance. These interventions would benefit from further, 
larger-scale studies, or more rigorous evaluation. The sharing of outcome measures across studies would also allow for improved future quantitative synthesis.

We found that research often focused on single centres, also noted by Dettrick et al. [23]. More work should be done to evaluate quality improvement at multiple centres in different settings, such as comparisons between rural and urban hospitals, and to investigate the impact of scaling up existing projects [12, 73]. This may need specific capacity building of public health programmes and clinical staff on research in LMICs and provision of grants to undertake context-specific projects on a range of interventions. Barriers and promoters were not assessed for statistical significance by any study, and multi-centre studies could enable such analysis and the provision of richer qualitative data. Generally, we would recommend that QI projects are rigorously evaluated and the experience documented or reported in peer-reviewed literature wherever feasible.

\section{Limitations of our study}

The heterogeneity of our study population, the interventions for quality improvement and multiple outcome measures were key challenges. Quality improvement approaches is an umbrella term for a variety of interventions, and though we cast a wider net, some studies may have been missed from our selection criteria, especially if they did not use the exact term. Many healthcare interventions in LMICs are community- rather than hospital-based, and care during pregnancy was not considered, so many interventions that may be useful in mitigating overall newborn morbidity and mortality were not eligible [74]. In general, many quality improvement projects do not progress to published literature, particularly small-scale projects, so publication bias is likely to be present. Unpublished ongoing initiatives ongoing in collaboration with WHO, UNICEF, USAID and others could not be included.

This study did not capture solely qualitative literature, and qualitative synthesis would be useful going forward, particularly for identifying barriers and promoters. There was generally little information on the methodology for identifying barriers and promoters in the included studies, which may mean that findings are not fully representative. The study method did not allow for collection of information relating to parental experience, which is a critical dimension of quality of care improvement. This study is also limited by only including studies published from 2000 onwards and published in English. We did not conduct overall outcome-specific assessments of quality of evidence with a tool such as GRADE. Finally, although we aimed to analyse with reference to wealth, rural/urban, and type of facility as measures of healthcare equity, data on financing of health settings were available for only a minority of the included papers, and many hospital settings were anonymised. As a result, these analyses were not undertaken.

\section{Conclusion}

Going forward, we recommend more rigorous evaluation of quality improvement in neonatal hospital care. Interventions are commonly at the meso level and educational in nature, and more focus is required around macro- and micro-level interventions; other study designs should be explored, with direct investigation of barriers and promoters. This should be linked to programmatic efforts where possible, in order to combine implementation and research. Small and sick hospitalised newborns in LMICs are a population at the highest risk-they should be one of the prime beneficiaries of quality of care interventions and investments. Targeted resources will be needed to strengthen human resource capabilities for implementation research into quality improvement for small and sick newborn care and to document outcomes, costs, and lessons learnt.

\section{Additional file}

Additional file 1: Pilot extraction worksheet. List of fields which were extracted from studies where available. (DOCX 109 kb)

\section{Abbreviations}

CLABSI: Central line-associated bloodstream infections; ICTRP: International Clinical Trials Registry Platform; KMC: Kangaroo Mother Care; LMICs: Low- and middle-income countries; NICU: Neonatal Intensive Care Unit; POINTS of Care: Educational package for control of pain, oxygenation, infection, nutrition, and temperature and to improve supportive care; QI: Quality improvement; SGA: Small for gestational age; STABLE: Sugar, temperature, airway, blood pressure, lab work, and emotional support; UNICEF: United Nations International Children's Emergency Fund; USAID: United States Agency for International Development; VAP: Ventilator-associated pneumonia; WHO: World Health Organization

\section{Acknowledgements}

We would like to thank Sufang Guo (UNICEF ROSA) for suggesting the grey literature studies and Hannah Leslie, Margaret Kruk and Anna Gage for allowing the use of their 'Synthesizing Improvement Approaches' schema. We also acknowledge the support and review from the following colleagues in the process of preparation of this paper: Dr. Stefan Swartling Peterson (UNICEF), Willibald Zeck (UNICEF) and Lily Kak (USAID). We would like to thank Lisa Amani and Jamila Adi for assisting with writing the review protocol.

\section{Funding}

This research received no specific grant from any funding agency, commercial or not-for-profit sectors. Logan Manikam is funded by a National Institute for Health Research (NIHR) Doctoral Research Fellowship (DRF-201407-005) and undertook an unpaid internship at UNICEF New York to complete this project. Mike English is supported by funding from The Wellcome Trust (\#097170) and Gina Murphy by funding from Health Systems Research Initiative (Department for International Development, UK (DFID), Economic and Social Research Council (ESRC), Medical Research Council (MRC) and Wellcome Trust, grant number MR/M015386/1. Pavani Ram and Susan Niermeyer are funded by USAID through their respective university roles. Nabila Zaka and Luwei Pearson are UNICEF staff based at the UNICEF New York headquarters. NIHR and all other funders had no role in the design, analysis or writing of this article. 


\section{Availability of data and materials}

All data generated or analysed during this study are included in this published article and its supplementary information files.

\section{Authors' contributions}

$\mathrm{NZ}$ and LM conceived of and designed the review. NZ, EA, LM, IN, and MA coordinated and undertook the screening and data extraction. All authors contributed equally to write the draft, read and approve the final manuscript.

\section{Ethics approval and consent to participate}

Not applicable.

\section{Consent for publication}

Not applicable.

\section{Competing interests}

The authors declare that they have no competing interests.

\section{Publisher's Note}

Springer Nature remains neutral with regard to jurisdictional claims in published maps and institutional affiliations.

\section{Author details}

'UNICEF New York, UNICEF House, 3 United Nations Plaza, New York, NY 10017, USA. ${ }^{2}$ King's College London GKT School of Medical Education, Guy's Campus, London SE1 1UL, UK. ${ }^{3}$ UCL Institute Epidemiology \& Healthcare, 1 19 Torrington Place, London WC1E 6BT, UK. ${ }^{4}$ Maternal, Adolescent, Reproductive and Child Health (MARCH) Centre and Department of Infectious Disease Epidemiology, London School of Hygiene and Tropical Medicine, Keppel Street, London WC1E 7HT, UK. ${ }^{5}$ Department of Epidemiology and Environmental Health, 237 Farber Hall, Buffalo, NY 14214-8001, USA. ${ }^{6}$ Office of Maternal and Child Health and Nutrition, USAID, Washington DC, USA. ${ }^{7}$ Centre for Tropical Medicine and Global Health, Nuffield Department of Medicine Research Building, University of Oxford, Old Road Campus, Roosevelt Drive, Headington, Oxford OX3 7FZ, UK. ${ }^{8}$ Section of Neonatology, University of Colorado School of Medicine, Aurora, CO 80045 USA

Received: 28 June 2017 Accepted: 16 January 2018 Published online: 25 January 2018

\section{References}

1. UNICEF. Levels \& trends in child mortality: report; 2017. p. 2017.

2. Lawn JE, Blencowe H, Oza S, You D, ACC L, Waiswa P, et al. Every newborn: progress, priorities, and potential beyond survival. Lancet. 2014;384:189-205.

3. Katz J, ACC L, Kozuki N, Lawn JE, Cousens S, Blencowe H, et al. Mortality risk in preterm and small-for-gestational-age infants in low-income and middleincome countries: a pooled country analysis. Lancet. 2013;382:417-25.

4. Blencowe H, Cousens S, Chou D, Oestergaard M, Say L, Moller A-B, et al. Born too soon: the global epidemiology of 15 million preterm births. Reprod Health. 2013;10(Suppl 1):S2. https://doi.org/10.1186/1742-4755-10-S1-S2.

5. Chiavaroli V, Marcovecchio MLML, De Giorgis T, Diesse L, Chiarelli F, Mohn A. Progression of cardio-metabolic risk factors in subjects born small and large for gestational age. PLoS One. 2014;9:e104278. https://doi.org/10.1371/journal. pone.0104278

6. Lundgren EM, Tuvemo T. Effects of being born small for gestational age on long-term intellectual performance. Best Pract Res Clin Endocrinol Metab. 2008:22:477-88. https://doi.org/10.1016/j.beem.2008.01.014

7. Seale AC, Blencowe H, Manu AA, Nair H, Bahl R, Qazi SA, et al. Estimates of possible severe bacterial infection in neonates in sub-Saharan Africa, south Asia, and Latin America for 2012: a systematic review and meta-analysis. Lancet Infect Dis. 2014;14:731-41.

8. Lawn JE, Lee ACC, Kinney M, Sibley L, Carlo WA, Paul VK, et al. Two million intrapartum-related stillbirths and neonatal deaths: where, why, and what can be done? In: International Journal of Gynecology and Obstetrics. 2009.

9. Ovretveit J, Appleby J. Does improving quality of care save money? 2009. https://doi.org/10.1136/bmi.b3678.

10. Kruk M, Gage A. Synthesizing improvement approaches. In: Lancet global health high quality health systems in the sustainable development goal era (HQSS) commission inception meeting; 2017.
11. Batalden PB, Davidoff F. What is "quality improvement" and how can it transform healthcare? Qual Saf Heal Care. 2007:16:2-3.

12. Bhutta ZA, Das JK, Bahl R, Lawn JE, Salam RA, Paul VK, et al. Can available interventions end preventable deaths in mothers, newborn babies, and stillbirths, and at what cost? Lancet. 2014;384:347-70.

13. Sohn AH, Garrett DO, Sinkowitz-Cochran RL, Grohskopf LA, Levine GL, Stover $\mathrm{BH}$, et al. Prevalence of nosocomial infections in neonatal intensive care unit patients: results from the first national point-prevalence survey. J Pediatr. 2001;139:821-7.

14. Won S-P, Chou H-C, Hsieh W-S, Chen C-Y, Huang S-M, Tsou K-I, et al. Handwashing program for the prevention of nosocomial infections in a neonatal intensive care unit. Infect Control Hosp Epidemiol. 2004;25:742-6.

15. Rasul $\mathrm{CH}$, Hassan MA, Habibullah M. Neonatal sepsis and use of antibiotic in a tertiary care hospital. Pakistan J Med Sci. 2007;23:78-81.

16. Engmann C, Wall S, Darmstadt G, Valsangkar B, Claeson M. Consensus on kangaroo mother care acceleration. Lancet. 2013;382:e26-e27.

17. Renfrew MJ, Craig D, Dyson L, McCormick F, Rice S, King SE, et al. Breastfeeding promotion for infants in neonatal units: a systematic review and economic analysis. Health Technol Assess. 2009;13:1-iv. https://doi.org/ 10.3310/hta13400

18. Vento M, Escobar J, Cernada M, Escrig R, Aguar M. The use and misuse of oxygen during the neonatal period. Clin Perinatol. 2012;39:165-76.

19. Cooper LG, Gooding JS, Gallagher J, Sternesky L, Ledsky R, Berns SD. Impact of a family-centered care initiative on NICU care, staff and families. J Perinatol. 2007;27:32-7.

20. Levin A. Humane neonatal care initiative. Acta Paediatr. 1999;88:353-5. https://doi.org/10.1111/j.1651-2227.1999.tb01118.x.

21. Taylor C, Gribble K, Sheehan A, Schmied V, Dykes F. Staff perceptions and experiences of implementing the baby friendly initiative in neonatal intensive care units in Australia. J Obstet Gynecol Neonatal Nurs. 2011;40:25-34.

22. Levin A. Are NICUs baby friendly or breastfeeding friendly? Birth. 2013;40:152-3.

23. Dettrick $Z$, Firth $S$, Jimenez Soto E. Do strategies to improve quality of maternal and child health care in lower and middle income countries lead to improved outcomes? A review of the evidence. PLoS One. 2013;8:e83070.

24. Althabe F, Bergel E, Cafferata ML, Gibbons L, Ciapponi A, Aleman A, et al. Strategies for improving the quality of health care in maternal and child health in low- and middle-income countries: an overview of systematic reviews. Paediatr Perinat Epidemiol. 2008:22:42-60.

25. Austin A, Langer A, Salam RA, Lassi ZS, Das JK, Bhutta ZA. Approaches to improve the quality of maternal and newborn health care: an overview of the evidence. Reprod Health. 2014;11(Suppl 2):S1. https://doi.org/10.1186/ 1742-4755-11-S2-S1.

26. The World Bank. Low \& middle income. 2017. http://data.worldbank.org/ income-level/low-and-middle-income. Accessed 1 Apr 2017.

27. Lawn JE, Mwansa-Kambafwile J, Horta BL, Barros FC, Cousens S. "Kangaroo mother care" to prevent neonatal deaths due to preterm birth complications (structured abstract). Int J Epidemiol. 2010;2 Supplement 1: i144-54. https://doi.org/10.1093/ije/dyq031.

28. Conde-Agudelo A, Belizán JM, Diaz-Rossello J. Kangaroo mother care to reduce morbidity and mortality in low birthweight infants. Cochrane Database Syst Rev. 2016;2016(8):CD002771.

29. National Institute of Clinical Excellence (NICE). NICE glossary. https://www. nice.org.uk/glossary. Accessed 1 Apr 2017.

30. Tuncalp. Quality of care for pregnant women and newborns - the WHO vision. World Health Organization; 2015.

31. Sterne JA, Hernán MA, Reeves BC, Savović J, Berkman ND, Viswanathan M, et al. ROBINS-l: a tool for assessing risk of bias in non-randomised studies of interventions. BMJ. 2016:14919. https://doi.org/10.1136/bmj.i4919.

32. Higgins J, Sterne J, Savović J, Page M, Hróbjartsson A, Boutron I, et al. A revised tool for assessing risk of bias in randomized trials. Cochrane Database Syst Rev. 2016;10(Supplement 1). https://sites.google.com/site/ riskofbiastool//welcome/rob-2-0-tool.

33. Rosenthal VD, Rodríguez-Calderón ME, Rodríguez-Ferrer M, Singhal T, Pawar M, Sobreyra-Oropeza M, et al. Findings of the International Nosocomial Infection Control Consortium (INICC), Part II: Impact of a Multidimensional Strategy to Reduce Ventilator-Associated Pneumonia in Neonatal Intensive Care Units in 10 Developing Countries. Infect Control Hosp Epidemiol. 2012; 33:696-703. https://doi.org/10.1086/666342

34. Rosenthal VD, Dueñas L, Sobreyra-Oropeza M, Ammar K, Navoa-Ng JA, De Casares ACB, et al. Findings of the International Nosocomial Infection Control Consortium (INICC), part III: effectiveness of a multidimensional 
infection control approach to reduce central line-associated bloodstream infections in the neonatal intensive care units of 4 developing countries. Infect Control Hosp Epidemiol 2013;34:229-237. https://doi.org/10.1086/ 669511

35. Srofenyoh E, Ivester T, Engmann C, Olufolabi A, Bookman L, Owen M. Advancing obstetric and neonatal care in a regional hospital in Ghana via continuous quality improvement. Int J Gynecol Obstet. 2012;116:17-21.

36. Agarwal R, Agarwal K, Acharya U, Christina P, Sreenivas V, Seetaraman S. Impact of simple interventions on neonatal mortality in a low-resource teaching hospital in India. J Perinatol. 2007;27:44-9. https://doi.org/10.1038/ sj.jp.7211620.

37. UNICEF. Special care newborn unit (SCANU): a major life saver. Bangladesh Ctry Off - Heal Sect. 2014; September 2014. https://www.unicef.org/ bangladesh/Scanu.pdf.

38. Crouse HL, Torres F, Vaides H, Walsh MT, Ishigami EM, Cruz AT, et al. Impact of an emergency triage assessment and treatment (ETAT)-based triage process in the paediatric emergency department of a Guatemalan public hospital. Paediatr Int Child Health. 2015;:2046905515Y0000000026. doi: https://doi.org/10.1179/2046905515Y.0000000026.

39. Gathara D, Opiyo N, Wagai J, Ntoburi S, Ayieko P, Opondo C, et al. Quality of hospital care for sick newborns and severely malnourished children in Kenya: a two-year descriptive study in 8 hospitals. BMC Health Serv Res. 2011;11:307. https://doi.org/10.1186/1472-6963-11-307.

40. Leng $H$, Wang $H$, Lin $B$, Cheng $G$, Wang L. Reducing transitional hypothermia in outborn very low birth weight infants. Neonatology. 2015: 109:31-6. https://doi.org/10.1159/000438743.

41. Mais A, Hajar F, Rajab M. A quality improvement program to reduce central line associated blood stream infections in neonates. Br J Med Med Res. 2015;7:638-46. https://doi.org/10.9734/BJMMR/2015/12976.

42. Namazzi G, Waiswa P, Nakakeeto M, Nakibuuka VK, Namutamba S, Najjemba $M$, et al. Strengthening health facilities for maternal and newborn care: experiences from rural eastern Uganda. Glob Health Action. 2015;8:1-8.

43. Pinto MCFG, Bueno AC, Vieira AA. Implementation of a protocol proposed by the Brazilian National Health Surveillance Agency for antibiotic use in very low birth weight infants. J Pediatr. 2013:89(5):450. https://doi.org/10. 1016/j.jped.2013.01.009.

44. Rahman AE, lqbal A, Hoque DM, Moinuddin M, Zaman SB, Rahman QS, et al. Managing neonatal and early childhood syndromic sepsis in sub-district hospitals in resource poor settings: improvement in quality of care through introduction of a package of interventions in rural Bangladesh. PLOS ONE. 2017;12:no pagination. http://journals.plos.org/plosone/article?id=10.1371/ journal.pone.0170267.

45. Salehi Z, Mokhtari Nouri J, Khademolhoseyni SM, Ebadi A. The effect of education and implementation of evidence-based nursing guidelines on infants' weight gaining in NICU. Glob J Health Sci. 2015;7:148-53. https:// doi.org/10.5539/gjhs.v7n2p148.

46. Zhou Q, Lee SK, Hu X, Jiang S, Chen C, Wang C, et al. Successful reduction in central line-associated bloodstream infections in a Chinese neonatal intensive care unit. Am J Infect Control. 2015;43:275-9. https://doi.org/10. 1016/j.ajic.2014.12.001.

47. dos Santos GC, de Lima LM, de Oliveira GB, de Souza AR, dos Freitas VS. Nursing intervention for pain control in newborns: effectiveness of nonpharmacological actions. J Nurs UFPE on line. 2015;9:8784-92.

48. Bastani F, Abadi TA, Haghani $\mathrm{H}$. Effect of family-centered care on improving parental satisfaction and reducing readmission among premature infants: a randomized controlled trial. J Clin Diagn Res. 2015;9:SC04-8. https://doi.org/ 10.7860/JCDR/2015/10356.5444

49. Z a B, Khan I, Salat S, Raza F, Ara H. Reducing length of stay in hospital for very low birthweight infants by involving mothers in a stepdown unit: an experience from Karachi (Pakistan). BMJ. 2004;329:1151-5. https://doi.org/10. 1136/bmj.329.7475.1151.

50. Clark M, Spry E, Daoh K, Baion D, Skordis-Worrall J. Reductions in inpatient mortality following interventions to improve emergency hospital care in Freetown, Sierra Leone. PLoS One. 2012;7(9):e41458.

51. Darmstadt GL, Ahmed A SMNU, Saha SK, K a CM a, M A A, Khatun M, et al. Infection control practices reduce nosocomial infection and mortality in preterm infants in Bangladesh. J Perinatol. 2005;25(5):331. https://doi.org/10. 1038/sj.jp.7211283.

52. Erdeve O, Arsan S, Yigit S, Armangil D, Atasay B, Korkmaz A. The impact of individual room on rehospitalization and health service utilization in preterms after discharge. Acta Paediatr Int J Paediatr. 2008;97:1351-1357.
53. Gilbert C, Darlow B, Zin A, Sivasubramaniam S, Shah S, Gianini N, et al. Educating neonatal nurses in Brazil: a before-and-after study with interrupted time series analysis. Neonatology. 2014;106:201-8.

54. Sethi A, Joshi M, Thukral A, Singh Dalal J, Kumar DA. A quality improvement initiative: improving exclusive breastfeeding rates of preterm neonates. Indian J Pediatr. 2017;84 April:322-5. https://doi.org/10.1007/s12098-0172306-4.

55. Soni A, Amin A, Patel D V, Fahey N, Shah N, Phatak AG, et al. The presence of physician champions improved kangaroo mother care in rural western India. Acta Paediatr Int J Paediatr 2016;105:e390-e395. https://doi.org/10. 1111/apa.13445.

56. Wrammert J, KC A, Ewald U, Malqvist M. Improved postnatal care is needed to maintain gains in neonatal survival after the implementation of the helping babies breathe initiative. Acta Paediatr. 2017;106(8):1280-5.

57. Zhou Q, Lee SK, Jiang S, Chen C, Kamaluddeen M, Hu X, et al. Efficacy of an infection control program in reducing ventilator-associated pneumonia in a Chinese neonatal intensive care unit. Am J Infect Control. 2013;41:1059-64. https://doi.org/10.1016/j.ajic.2013.06.007.

58. Cavicchiolo ME, Lanzoni P, Wingi MO, Pizzol D, Daverio M, Da Dalt L, et al. Reduced neonatal mortality in a regional hospital in Mozambique linked to a quality improvement intervention. BMC Pregnancy Childbirth. 2016;16:366. https://bmcpregnancychildbirth.biomedcentral.com/articles/10.1186/s12884016-1170-y.

59. Ramaswamy R, Iracane S, Srofenyoh E, Bryce F, Floyd L, Kallam B, et al. Transforming maternal and neonatal outcomes in tertiary hospitals in Ghana: an integrated approach for systems change. J Obstet Gynaecol Canada. 2015;37:905-14. http://www.jogc.com/article/S1701-2163(16)300299/fulltext.

60. Yawson AE, Awoonor-Williams JK, Sagoe-Moses I, Aboagye PK, Yawson AO, Senaya LK, et al. Bottleneck analysis approach to accelerate newborn care services in two regions in Ghana: implications for national newborn care. Public Health. 2016;141:245-54.

61. Soni A, Amin A, Patel DV, Fahey N, Shah N, Phatak AG, et al. The presence of physician champions improved kangaroo mother care in rural western India. Acta Paediatr Int J Paediatr. 2016;105:e390-5. http://onlinelibrary.wiley. com/doi/10.1111/apa.13445/abstract.

62. Moxon SG, Lawn JE, Dickson KE, Simen-Kapeu A, Gupta G, Deorari A, et al. Inpatient care of small and sick newborns: a multi-country analysis of health system bottlenecks and potential solutions. BMC Pregnancy Childbirth. 2015;15(Suppl 2):S7. https://doi.org/10.1186/1471-2393-15-S2-S7.

63. Simen-Kapeu A, Seale AC, Wall S, Nyange C, Qazi SA, Moxon SG, et al. Treatment of neonatal infections: a multi-country analysis of health system bottlenecks and potential solutions. BMC Pregnancy Childbirth. 2015;15 Suppl 2:S6. https://doi.org/10.1186/1471-2393-15-S2-S6.

64. Opiyo N, English M. In-service training for health professionals to improve care of seriously ill newborns and children in low-income countries. The Cochrane database of systematic reviews. 2015;5:CD007071.

65. Schouten LMT, Hulscher MEJL, Everdingen JJE V., Huijsman R, Grol RPTM. Evidence for the impact of quality improvement collaboratives: systematic review. BMJ 2008:336:1491-1494. doi:https://doi.org/10.1136/bmj.39570. 749884.BE.

66. Shah AJ, Mulla SA, Revdiwala SB. Neonatal sepsis: high antibiotic resistance of the bacterial pathogens in a neonatal intensive care unit of a tertiary care hospital. J Clin Neonatol. 2012;1:72-5.

67. Darlow BA, Zin AA, Beecroft G, Moreira MEL, Gilbert CE. Capacity building of nurses providing neonatal care in Rio de Janeiro, Brazil: methods for the POINTS of care project to enhance nursing education and reduce adverse neonatal outcomes. BMC Nurs. 2012;11:3. https://doi.org/10.1186/1472-6955$11-3$.

68. United Nations. Saving lives protecting futures: progress report on the global strategy for women's and children's health 2010-2015. 2015. http:// www.everywomaneverychild.org/images/EWEC_Progress_Report_FINAL_3. pdf. Accessed 1 Apr 2017.

69. UNICEF, WHO. Every newborn: an action plan to end preventable deaths. 2014

70. Chan G, Bergelson I, Smith ER, Skotnes T, Wall S. Barriers and enablers of kangaroo mother care implementation from a health systems perspective: a systematic review. Health Policy Plan. 2017:1-10.

71. Pattinson R, Kerber K, Waiswa P, Day LT, Mussell F, Asiruddin S, et al. Perinatal mortality audit: counting, accountability, and overcoming challenges in scaling up in low- and middle-income countries. In: International journal of gynecology and obstetrics; 2009. 
72. Bandali S, Thomas C, Hukin E, Matthews Z, Mathai M, Ramachandran Dilip T, et al. Maternal death surveillance and response systems in driving accountability and influencing change. Int J Gynecol Obstet. 2016;135:36571. https://doi.org/10.1016/j.ijgo.2016.10.002.

73. KC A, Bergström A, Chaulagain D, Brunell O, Ewald U, Gurung A, et al. Scaling up quality improvement intervention for perinatal care in Nepal (NePeriQIP); study protocol of a cluster randomised trial. BMJ Glob Heal. 2017;2::e000497.

74. Lawn JE, Bhutta ZA, Wall SN, Peterson S, Daviaud E. Cadres, content and costs for community based care for mothers and newborns from seven countries: implications for universal health coverage. Health Policy Plan. 2017;32:11-5.

75. Moher D, Liberati A, Tetzlaff JAD. PRISMA 2009 flow diagram. The PRISMA statement. 2009;6:1000097.

\section{Submit your next manuscript to BioMed Central} and we will help you at every step:

- We accept pre-submission inquiries

- Our selector tool helps you to find the most relevant journal

- We provide round the clock customer support

- Convenient online submission

- Thorough peer review

- Inclusion in PubMed and all major indexing services

- Maximum visibility for your research

Submit your manuscript at www.biomedcentral.com/submit 